\title{
Measuring the Work Function at a Nanobelt Tip and at a Nanoparticle Surface
}

\author{
Xuedong Bai, ${ }^{\dagger, \ddagger}$ Ee Ge Wang, ${ }^{\ddagger}$ Puxian Gao, ${ }^{\dagger}$ and Zhong Lin Wang ${ }^{\star}, \dagger$ \\ School of Materials Science and Engineering, Georgia Institute of Technology, \\ Atlanta Georgia 30332-0245, and International Center for Quantum Structures \\ and State Key Laboratory for Surface Physics, Institute of Physics, \\ Chinese Academy of Sciences, Beijing 100080, China
}

Received May 26, 2003; Revised Manuscript Received June 11, 2003

\begin{abstract}
The work function at the tips of individual $\mathrm{ZnO}$ nanobelts has been measured by an electromechanical resonant method using in-situ transmission electron microscopy. The work function of the $\mathrm{ZnO}$ nanobelts is $\sim 5.2 \mathrm{eV}$, which shows no significant dependence on the geometrical size. Using a $\mathrm{ZnO}$ nanobelt as a carrier beam, the work function of a single nanoparticle of a different material has also been measured.
\end{abstract}

One-dimensional oxide nanostructures are stimulating a great deal of interest because of their novel properties, diverse functionalities, surface cleanliness, and chemical/thermal stability. ${ }^{1-9}$ Among those oxides, zinc oxide $(\mathrm{ZnO})$ is a semiconductor with a wide band gap of $3.37 \mathrm{eV}$ and a large exciton binding energy of $60 \mathrm{meV}$, which is suitable for short-wavelength optoelectronic devices at room temperature. $\mathrm{ZnO}$ nanobelts and nanowire have shown interesting electronic, chemical, photoluminescence, and lasing properties. ${ }^{4,10-12}$ The field-emission property for well-aligned $\mathrm{ZnO}$ nanowire arrays has been reported, ${ }^{13}$ demonstrating a promising application of semiconductor nanowires as field emitters for flat panel display.

Following the Fowler-Nordheim $(\mathrm{F}-\mathrm{N})$ theory, ${ }^{14}$ an important physical quantity in electron field emission is the surface work function, which is well documented for elemental materials. For the emitters such as $\mathrm{ZnO}$ nanowire arrays, most of the electrons are emitted from tips of the nanowires, and it is the local work function that matters to the properties of the field emission. However, the work function measured based on the $\mathrm{F}-\mathrm{N}$ theory is an average over the large scale of emitting materials. So it is necessary to measure the local work function at the tip of individual emitter. Gao et al. ${ }^{15}$ have measured the work function at the tips of carbon nanotubes by an in-situ transmission electron microscopy (TEM) technique. The measurement relies on the mechanical resonance of the carbon nanotube induced

\footnotetext{
* Corresponding author: E-mail: zhong.wang@mse.gatech.edu

Georgia Institute of Technology.

$\doteqdot$ Chinese Academy of Sciences.
}

by an externally applied oscillating voltage with tunable frequency. ${ }^{16}$ In this paper, we report the measurements of work functions at the tips of individual $\mathrm{ZnO}$ nanobelts using the in-situ TEM technique. The work function at the tips of the $\mathrm{ZnO}$ nanobelts is $\sim 5.2 \mathrm{eV}$, which shows little dependence on their dimension. This result provides an experimental basis for the work function of $\mathrm{ZnO}$ nanostructures and is important for understanding their field emission characteristics.

Figure 1a is a typical scanning electron microscopy (SEM) image of the as-prepared $\mathrm{ZnO}$ nanobelts produced at a high yield. The lengths of the $\mathrm{ZnO}$ nanobelts are in the range of several tens to several hundreds of micrometers. A transmission electron microscope (TEM) image, as shown in Figure $1 \mathrm{~b}$, reveals the high structural uniformity of the $\mathrm{ZnO}$ nanobelts. The selected area electron diffraction pattern (inset in Figure 1b) indicates that the nanobelt grows along [0001] and is enclosed by $\pm(2 \overline{1} \overline{1} 0)$ and $\pm(01 \overline{1} 0)$ facets. ${ }^{1}$

The measurement of work function at the tip of a single $\mathrm{ZnO}$ nanobelt was carried out in-situ at $200 \mathrm{kV}$ in a Hitachi HF-2000 FEG TEM. A specimen holder was built for applying a voltage across a nanobelt and its counter gold electrode, as reported in detail elsewhere. ${ }^{17,18}$ The nanobelts to be used for measurements are directly imaged under TEM. Figure 2 is a setup for work function measurement. One end of the nanobelt was electrically attached to a gold wire, and the other end faces directly against the gold ball. Because of the difference in the surface work function between the $\mathrm{ZnO}$ nanobelt and the counter $\mathrm{Au}$ ball, a static charge $Q_{0}$ exists at the tip of the nanobelt to balance this potential difference. ${ }^{19}$ The magnitude of $Q_{0}$ is proportional to the 


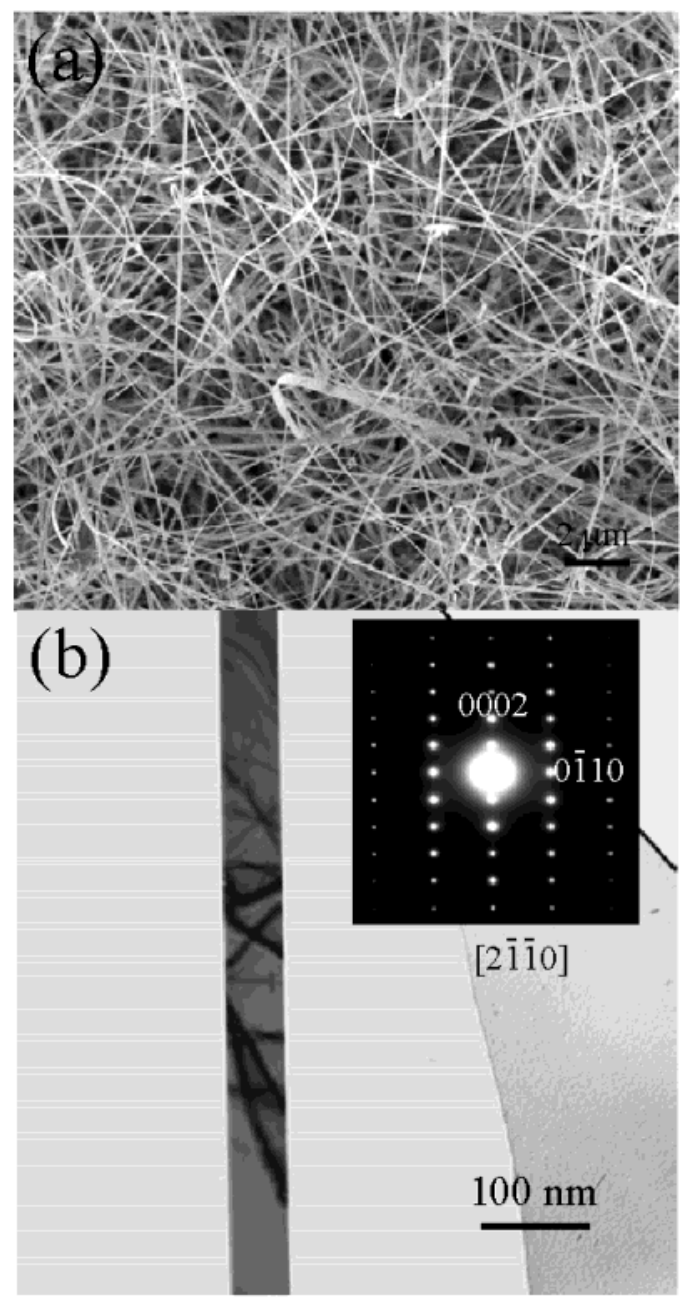

Figure 1. (a) SEM image of the as-grown $\mathrm{ZnO}$ nanobelts. (b) TEM image of the $\mathrm{ZnO}$ nanobelt; the inset is its electron diffraction pattern, which indicates the nanobelt growing along [0001].

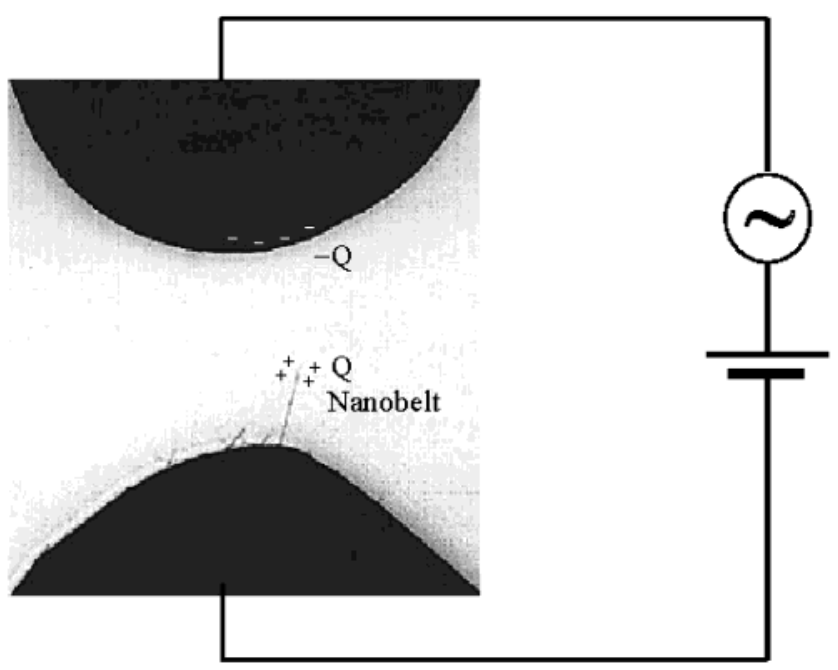

Figure 2. Experimental set up for measuring the work function at the tip of a $\mathrm{ZnO}$ nanobelt.

difference between work function of the nanobelt tip (NBT) and the Au electrode, $Q_{0}=\alpha\left(\phi_{\mathrm{Au}}-\phi_{\mathrm{NBT}}\right)$, where $\alpha$ is related to the geometry and distance between the nanobelt and the electrode.

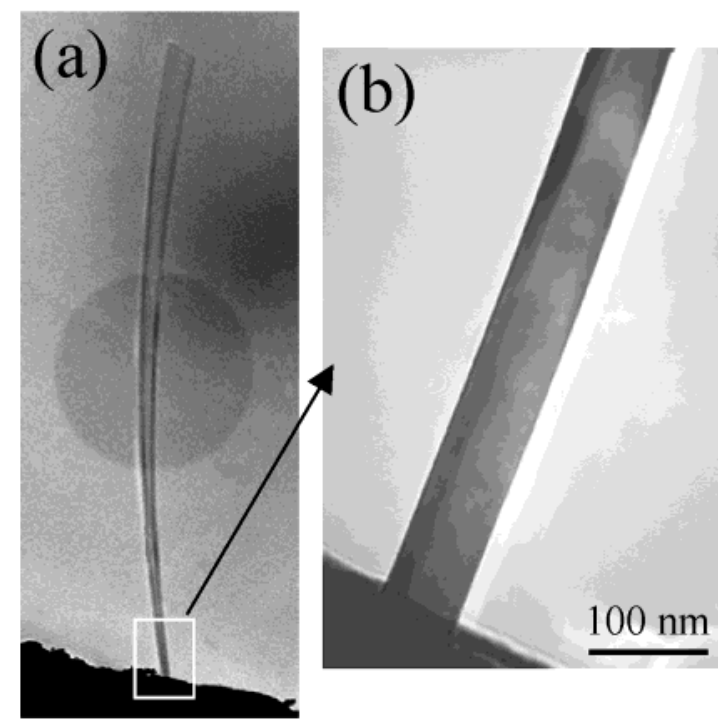

Figure 3. (a) TEM image of a fixed $\mathrm{ZnO}$ nanobelt at mechanical resonance as induced by an oscillating voltage at $f=0.576 \mathrm{MHz}$ and $V_{\mathrm{ac}}=3.0 \mathrm{~V}$. (b) TEM image recorded from the root of the nanobelt, showing the projected geometry of the nanobelt.

The measurement is based on the mechanical resonance of the nanobelt induced by an externally alternative electric field. Figure $3 \mathrm{a}$ shows the attached $\mathrm{ZnO}$ nanobelt can be regarded as a vibration cantilever clamped at one end. Experimentally, a constant voltage $V_{\mathrm{dc}}$ and an oscillating voltage $V_{\mathrm{ac}} \cos 2 \pi f t$ are applied to the nanobelt (Figure 2), where $f$ is the frequency and $V_{\text {ac }}$ is the amplitude. Thus, the total induced charge on the nanobelt is

$$
Q=Q_{0}+\alpha e\left(V_{\mathrm{dc}}+V_{\mathrm{ac}} \cos 2 \pi f t\right)
$$

The force acting on the nanobelt is proportional to the square of the total charge on the nanobelt

$$
\begin{aligned}
F= & \beta\left[Q_{0}+\alpha e\left(V_{\mathrm{dc}}+V_{\mathrm{ac}} \cos 2 \pi \mathrm{ft}\right)\right]^{2} \\
= & \alpha^{2} \beta\left\{\left[\left(\phi_{\mathrm{Au}}-\phi_{\mathrm{NBT}}+e V_{\mathrm{dc}}\right)^{2}+e^{2} V_{\mathrm{ac}}^{2} / 2\right]+\right. \\
& \left.2 e V_{\mathrm{ac}}\left(\phi_{\mathrm{Au}}-\phi_{\mathrm{NBT}}+e V_{\mathrm{dc}}\right) \cos 2 \pi f t+e^{2} V_{\mathrm{ac}}{ }^{2} / 2 \cos 4 \pi f t\right\}
\end{aligned}
$$

where $\beta$ is a proportional constant. In eq 2 , the first term is constant and it causes a static deflection of the $\mathrm{ZnO}$ nanobelt. The second term is a linear term, and the resonance occurs if the applied frequency $f$ is tuned to the intrinsic mechanical resonance frequency $f_{0}$ of the $\mathrm{ZnO}$ nanobelt, as shown in Figure $3 \mathrm{a}$. According to vibration theory and considering the rectangular cross-section of the nanobelt, ${ }^{20}$ mechanical resonance could be along the thickness or width direction at frequencies given by

$$
\begin{gathered}
f_{T}=\frac{1.875^{2} T}{8 \pi L^{2}} \sqrt{\frac{E_{T}}{3 \rho}} \\
f_{W}=\frac{1.875^{2} W}{8 \pi L^{2}} \sqrt{\frac{E_{W}}{3 \rho}}
\end{gathered}
$$




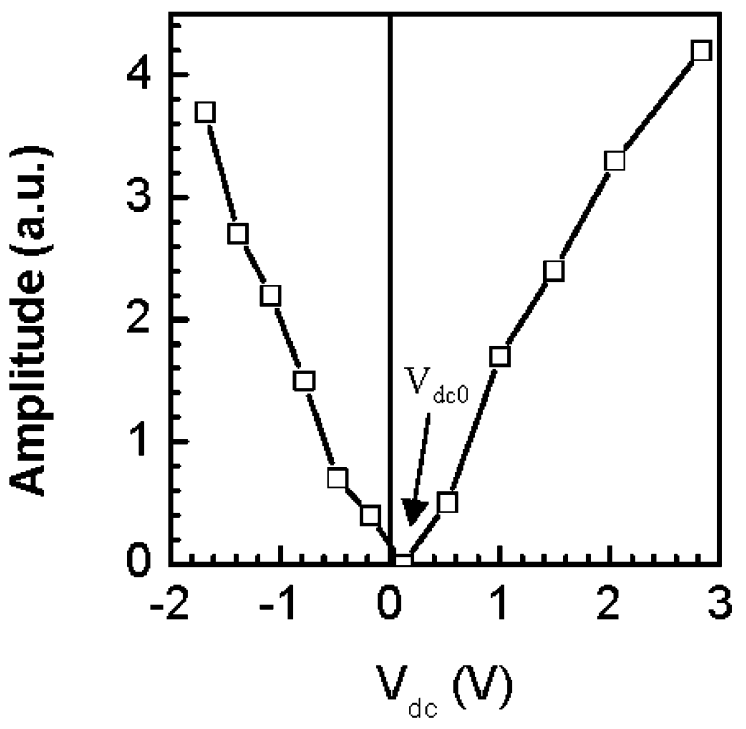

Figure 4. Plot of vibration amplitude of a $\mathrm{ZnO}$ nanobelt as a function of the applied direct current voltage, from which the offset voltage $V_{\mathrm{dc} 0}=0.12 \mathrm{~V}$.

respectively, where $T$ is the thickness of the nanobelt, $W$ its width, $L$ its length, $\rho$ the volume density, and $E$ the bending modulus of the nanotube. The last term in eq 2 is the second harmonics. The most important result of eq 2 is that, for the linear term, the resonance amplitude $A$ of the nanobelt is proportional to $V_{\mathrm{ac}}\left(\phi_{\mathrm{Au}}-\phi_{\mathrm{NBT}}+e V_{\mathrm{dc}}\right)$.

The principle of the measurement is as follows. We first set $V_{\mathrm{dc}}=0$ and tune the frequency $f$ to get the mechanical resonance induced by the applied oscillating field. Second, under the resonance condition of keeping $f=f_{0}$ and $V_{\mathrm{ac}}$ constant, slowly change the magnitude of $V_{\text {dc }}$ from zero to a value $V_{\mathrm{dc} 0}$ that satisfies $\phi_{\mathrm{Au}}-\phi_{\mathrm{NBT}}+\mathrm{eV}_{\mathrm{dc} 0}=0$. At this moment, the resonance amplitude $A$ becomes zero although the external AC voltage is still in effect. Therefore, the work function at the tip of $\mathrm{ZnO}$ nanobelt is $\phi_{\mathrm{NBT}}=\phi_{\mathrm{Au}}+e V_{\mathrm{dc} 0}$, while $\phi_{\mathrm{Au}}=5.1 \mathrm{eV} .{ }^{21}$ Figure 4 is a plot of the vibration amplitude $A$ of the nanobelt as a function of the applied direct current voltage $V_{\mathrm{dc}}$, from which the value for $V_{\mathrm{dc} 0}$ is determined. The dual-mode resonance of the nanobelts has been observed experimentally, as defined by eqs $3 a$ and $3 b .^{22}$ It is important to point out that it does not matter which resonance direction/frequency one chooses, either along the thickness or width direction, the measured $V_{\mathrm{dc} 0}$ remains the same.

To investigate the dependence of the work function of $\mathrm{ZnO}$ nanobelts on the size of their cross-section, i.e., the width $W$ and thickness $T$ of the nanobelts, we measured the geometrical size of the nanobelts from which the work function was measured. Using the projected dimension measured from the TEM image and the orientated angle determined from the corresponding electron diffraction pattern, the geometrical parameters of the nanobelts are obtained..$^{22}$ The experimental results are summarized in Table 1. The work function at the tip of the $\mathrm{ZnO}$ nanobelt is around $5.2 \mathrm{eV}$ and shows no significant dependence on the crosssection size of the $\mathrm{ZnO}$ nanobelts.
Table 1. Work Functions of the $\mathrm{ZnO}$ Nanobelts with Different Cross-Section Size

\begin{tabular}{cccc}
\hline nanobelt & width W $(\mathrm{nm})$ & thickness $\mathrm{T}(\mathrm{nm})$ & work function $\phi(\mathrm{eV})$ \\
\hline 1 & 78 & 43 & 5.22 \\
2 & 51 & 39 & 5.23 \\
3 & 55 & 35 & 5.18 \\
4 & 41 & 20 & 5.16 \\
5 & 34 & 20 & 5.26
\end{tabular}

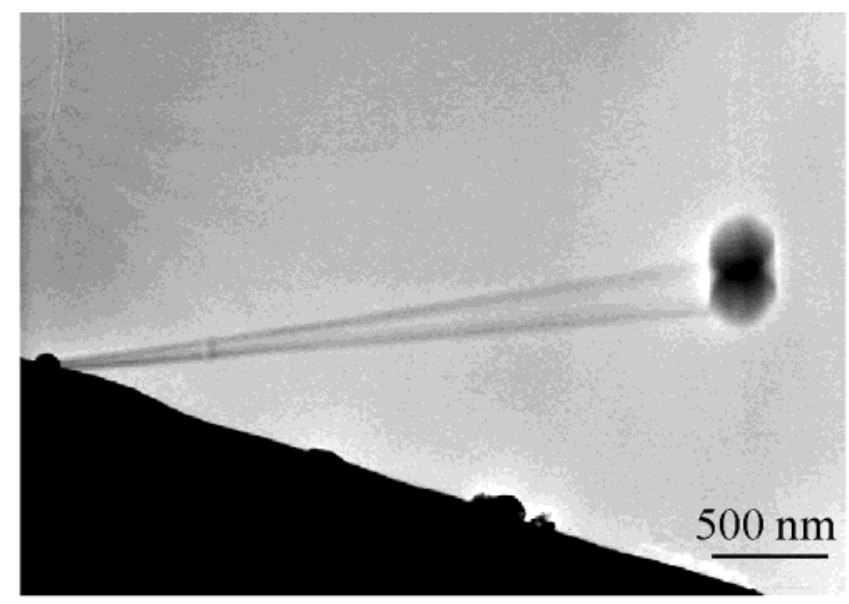

Figure 5. Measurement the work function of an attached carbon particle on the tip of a $\mathrm{ZnO}$ nanobelt at $f=0.349 \mathrm{MHz}$ and $V_{\mathrm{ac}}=$ $9.0 \mathrm{~V}$.

To ensure the accuracy of the measurements, the resonance stability and frequency drift of the $\mathrm{ZnO}$ nanobelts have been examined prior to and post measurement so that the change in vibration amplitude is solely attributed to the effect of $V_{\mathrm{dc}}$. The sensitivity of this measurement is excellent because the full width at half-maximum for the resonance peak is $\Delta f / f_{0}=0.2 \%$. Furthermore, the influence of surface condition on work function is important. In our experiments, the surfaces of the $\mathrm{ZnO}$ nanobelts are clean, atomically sharp, and without any sheathed amorphous phase. ${ }^{1}$

This resonant method can also be applied to measure the work functions of other materials, such as the case shown in Figure 5. A carbon particle of diameter $\sim 300 \mathrm{~nm}$ is attached at the tip of a nanobelt. Since the induced charges are accumulated mainly at the surface of the carbon particle, the measured work function should be for the surface of the particle. Using the same technique as demonstrated above, the mechanical resonance of the nanobelt gives a work function of $5.05 \mathrm{eV}$, which is consistent with that of carbon $\left(\phi_{\mathrm{C}}=5.0 \mathrm{eV}\right) .{ }^{23}$ This demonstrates a technique for measuring the work function of a single nanoparticle.

In conclusion, the work functions at the tips of individual $\mathrm{ZnO}$ nanobelts have been measured by an electromechanical resonant method using in-situ TEM. The work function at the tip of a $\mathrm{ZnO}$ nanobelt is $\sim 5.2 \mathrm{eV}$, which shows little dependence on the geometrical size. This quantity is important in the interpretation of the field emission properties of nanobelts. Using a $\mathrm{ZnO}$ nanobelt as a carrier beam, the work functions of a nanoparticle of a different material on the tip of the nanobelt has also been measured. 
Experimental Section. The synthesis of $\mathrm{ZnO}$ nanobelts is based on thermal evaporation of source materials under controlled conditions without the presence of catalyst. The commercial $\mathrm{ZnO}$ powder (purity: $99.99 \%$; $\mathrm{mp} 1975^{\circ} \mathrm{C}$ ) was loaded on an alumina boat and positioned at the center of an alumina tube inside a high-temperature furnace. After evacuating the tube to $2 \times 10^{-3}$ Torr, thermal evaporation was conducted at $1350^{\circ} \mathrm{C}$ for $1 \mathrm{~h}$ under the pressure of 200300 Torr and Ar carrier gas flow rate of $50 \mathrm{sccm}$. During evaporation, the products were deposited onto an alumina plate placed at the downstream of the alumina tube. The local growth temperature was $\sim 800{ }^{\circ} \mathrm{C}$.

Acknowledgment. Thanks to the support from the NSF of China Grant No. 10134030 and US NSF, and the Georgia Tech Electron Microscopy Center for providing the research facility.

\section{References}

(1) Pan, Z. W.; Dai, Z. R.; Wang, Z. L. Science 2001, 291, 1947.

(2) Puxian Gao, Wang, Z. L. J. Phys. Chem. B 2002, 106, 12653.

(3) Pan, Z. W.; Dai, Z. R.; Wang, Z. L. Appl. Phys. Lett. 2002, 80, 309.

(4) Huang, M. H.; Mao, S.; Feick, H.; Yan, H. Q.; Wu, Y. Y.; Kind, H.; Webber, E.; Russo, R.; Yang, P. D. Science 2001, 292, 1897.

(5) Xia, Y.; Yang, P.; Sun, Y.; Wu, Y.; Mayers, B.; Gates, B.; Yin, Y.; Kim, F.; Yan, H. Adv. Mater. 2003, 15, 353.

(6) Krumeich, F.; Muhr, H. J.; Niederberger, M.; Bieri, F.; Schnyder, B.; Nesper, R. J. Am. Chem. Soc. 1999, 121, 8324
(7) Choi, Y. C.; Kim, W. S.; Park, Y. S.; Lee, S. M.; Bae, D. J.; Lee, Y. H.; Park, G. S.; Choi, W. B.; Lee, N. S.; Kim, J. M. Adv. Mater. 2000, 12, 746.

(8) Adachi, M.; Harada, T.; Harada, M. Langmuir 1999, 15, 7097.

(9) Satishkumar, B. C.; Govindaraj, A.; Vogel, E. M.; Basumallick, L.; Rao, C. N. R. J. Mater. Res. 1997, 12, 604.

(10) Arnold, M. S.; Avouris, Ph.; Pan, Z. W.; Wang, Z. L. J. Phys. Chem. $B$ 2003, 107, 659 .

(11) Comini, E.; Faglia, G.; Sberveglieri, G.; Pan, Z. W.; Wang, Z. L. Appl. Phys. Lett. 2002, 81, 1869.

(12) Kong, Y. C.; Yu, D. P.; Zhang, B.; Fang, W.; Feng, S. Q. Appl. Phys. Lett. 2001, 78, 407.

(13) Lee, C. J.; Lee, T. J.; Lyu, S. C.; Zhang, Y.; Ruh, H.; Lee, H. J. Appl. Phy. Lett. 2002, 81, 3646.

(14) Fowler, R. H.; Nordheim, L. W. Proc. R. Soc. London, Ser. A 1928, 119, 173.

(15) Gao, R.; Pan, Z.; Wang, Z. L. Appl. Phys. Lett. 2001, 78, 1757.

(16) Poncharal, P.; Wang, Z. L.; Ugarte, D.; de Heer, W. A. Science 1999, $283,1513$.

(17) Wang, Z. L.; Poncharal, P.; de Heer, W. A. Pure Appl. Chem. 2000, 72, 209.

(18) Wang, Z. L.; Poncharal, P.; de Heer, W. A. Microsc. Microanal. 2000, 6, 224

(19) Lord Kelvin, Philos. Mag. 1898, 46, 82.

(20) Meirovich, L. Elements of Vibration Analysis; McGraw-Hill: New York, 1986.

(21) Eastman, D. E. Phys. Rev. B 1970, 2, 1.

(22) Bai, X. D.; Wang, E. G.; Gao, P. X.; Wang, Z. L. Appl. Phys. Lett., in press.

(23) Robrieux, B.; Faure, R.; Dussaulcy, J. P. C. R. Seances Acad. Sci. Ser. B 1974, 278, 659 .

NL034342P 